\title{
Reduced Complexity Calibration of MEMS IMUs
}

\author{
Emil Fresk, Sina Sharif Mansouri, Christoforos Kanellakis, Erik Halén and George Nikolakopoulos
}

\begin{abstract}
In this article a reduced complexity calibration method for Micro-Electro-Mechanical Systems (MEMS) Inertial Measurement Units (IMUs) will be presented, which does not need the rotating reference tables, commonly used in the gyroscope calibration. As it will be presented, in the proposed novel scheme fixed angle rotations have been utilized to observe the integral of the gyroscope signals to find the corresponding sensitivity, axis misalignment and acceleration sensitivity matrices. This appraoch has the significant merit of high norm accuracy, easiness of use, low cost and simplicity in construction, thus allowing anyone with a basic electronics knowledge to calibrate an IMU.
\end{abstract}

\section{INTRODUCTION}

IMUs consist of a well mastered technology in localization nowadays, with a huge number of massive application oriented utilizations, e.g. in mobile phones. IMUs also form the corner stone in the localization for robotic applications, with a profound variety in applications, spanning from underwater, to ground, aerial and space robotics. Their operation is based on fundamental data fusion, in most of the cases, applied on data streams from an accelerometer and gyroscope combo, while sometimes magnetometers are also utilized.

In outdoors applications, the Global Positioning System (GPS) is the first addressed solution for providing the necessary localization information, while for the case of indoors applications, IMUs are one of the most utilized short-term localization systems. Due to their importance, it is straight forward that the better their accurate operation is, the better positioning and levels of autonomy can be reached, and this is why multiple schemes have been appeared in the related literature for improving the data fusion and correspondingly the resulting positioning information.

Towards this direction, the IMU kinematic approaches are using estimators that are able to estimate the bias but not the corresponding gain of the IMU [1], with typical algorithmic implementations to be the ones in [2], [3], while [4], [5] presents the usage in visual inertial frameworks. An additional problem to the estimation schemes, based on IMUs, is the fact that the resulting axis misalignment, even only a few degrees, will be integrated and amplified in the estimation process with a corresponding degradation of the estimation results due to the integrating nature of the estimators. The last step in the IMU calibration is the correction of the position $R$ of the IMU, with respect to the

This work has received funding from the European Unions Horizon 2020 Research and Innovation Programme under the Grant Agreement No.644128, AEROWORKS

The authors are with the Department of Computer Science, Electrical and Space Engineering at Luleå University of Technology, SE-97187, Luleå, Sweden.

Corresponding Author's E-mail: emil.fresk@ltu.se (ㅇ)2017 IEEE
Center of Gravity (CoG). Towards this direction, multiple approaches have been also presented, such as the ones in [6], however still these are suffering from an increased cost and difficulties in their applicability.

The novelty of this article, stems from the utilization of fixed angle rotations to calibrate the gyro, without the need for a rotating table calibration. As it will be presented in the corresponding experimental results, this approach has the merit of significantly reducing the sensitivity errors, the axis misalignment and the bias errors of the IMU's output. The proposed novel scheme for the IMU calibration will be accompanied by the proposal of a novel calibration rig that also needs to be utilized.

The rest of the article is structured as it follows. In Section II-A the fundamental modeling behind the IMU operation is presented, while in Section III the proposed calibration methodology is being established. In Section IV multiple experimental results that prove the efficacy of the proposed scheme are presented, while the conclusions are drawn in the last Section V.

\section{A. Nomenclature}

Scalars are defined as non-bold characters, such as $s$ or $S$, while vectors are bold lower-case letters, such as $\boldsymbol{v}$ and finally matrices are bold upper-case letters, such as $\boldsymbol{A}$.

The coordinate frames in this article are defined as: 1) the world frame $\mathcal{W}$, and 2) the IMU frame $\mathcal{I}$, where, e.g. $\boldsymbol{v}_{\mathcal{W}}$ is a vector in the world frame.

The rotation matrices are following the passive notation that implies that the rotation matrix $\boldsymbol{R}_{\mathcal{G} \mathcal{L}}$ is a Local-to-Global rotation made in a right-handed coordinate system, implying $\boldsymbol{v}_{\mathcal{G}}=\boldsymbol{R}_{\mathcal{G} \mathcal{L}} \boldsymbol{v}_{\mathcal{L}}$.

\section{THEORY OF OPERATION}

\section{A. Inertial Measurement Unit}

The utilized sensor is the one of the MEMS IMU, more specifically the MEMS gyroscope and accelerometer due to their small size, low cost and high availability. These sensors do however suffer from several non-ideal effects, such as gain, bias and axis misalignment errors, which are a combination off static and varying errors. For the accelerometer and gyroscope these errors can be modeled in the standard way as in [7], [8]. More specifically, the accelerometer can be expressed as:

$$
\begin{aligned}
\boldsymbol{a}_{m} & =\boldsymbol{T}_{a} \boldsymbol{a}_{s}+\boldsymbol{a}_{b}+\boldsymbol{a}_{n} \\
\boldsymbol{a}_{s} & =\boldsymbol{a}_{t r u e}+\boldsymbol{R}_{\mathcal{W I}}^{T} \boldsymbol{g}_{\mathcal{W}} \\
\dot{\boldsymbol{a}}_{b} & =\boldsymbol{a}_{b n},
\end{aligned}
$$


with $\boldsymbol{T}_{a} \in \mathbb{R}^{3 \times 3}$ to be the transform matrix, where each row contains the corresponding sensitivity and axis misalignment as:

$$
\boldsymbol{t}_{i, a}=s_{i, a} \boldsymbol{u}_{i, a}^{T}
$$

where $s_{i, a} \in \mathbb{R}^{+}$is the accelerometer sensitivity and $\boldsymbol{u}_{i, a} \in$ $\mathbb{S}^{2}$ is the sensing direction of the $i^{t h}$ axis. While, $\boldsymbol{a}_{b} \in \mathbb{R}^{3}$ is the accelerometer bias, $\boldsymbol{a}_{n} \sim \mathcal{N}\left(0, \sigma_{a n}^{2}\right), \boldsymbol{a}_{b n} \sim \mathcal{N}\left(0, \sigma_{a b n}^{2}\right)$ denote the measurement and driving bias noise respectively, and $\boldsymbol{R}_{\mathcal{W I}}^{T} \boldsymbol{g}_{\mathcal{W}} \in \mathbb{R}^{3}$ is the gravity vector in the IMU frame. For the case of gyroscope, the errors can be defined as following:

$$
\begin{aligned}
\boldsymbol{\omega}_{m} & =\boldsymbol{T}_{\omega} \boldsymbol{\omega}_{\text {true }}+\boldsymbol{T}_{s} \boldsymbol{a}_{s}+\boldsymbol{\omega}_{b}+\boldsymbol{\omega}_{n} \\
\dot{\boldsymbol{\omega}}_{b} & =\boldsymbol{\omega}_{b n},
\end{aligned}
$$

where $\boldsymbol{T}_{\omega} \in \mathbb{R}^{3 \times 3}$ is the transform matrix containing the gain and the misalignment errors, similarly as in the accelerometer case, $\boldsymbol{T}_{s} \in \mathbb{R}^{3 \times 3}$ is the transform matrix containing acceleration sensitivity, and $\boldsymbol{\omega}_{n} \sim$ $\mathcal{N}\left(0, \sigma_{\omega n}^{2}\right), \boldsymbol{\omega}_{b n} \sim \mathcal{N}\left(0, \sigma_{\omega b n}^{2}\right)$ denotes measurement and driving bias noise respectively.

\section{MethodolOGY}

\section{A. Reference vectors}

In the case of the accelerometer, when no accelerations are exerted, equation (2) shows that the sensor is only measuring gravity, which is a known value. However, as the orientation of the sensor is unknown, only the norm of $\boldsymbol{a}_{s}$ is known, a fact that is common practice when calibrating MEMS accelerometers [9] and does not pose any issues with the calibration.

At this point it should be highlighted that when calibrating the gyroscope, there is no common angular rate reference, of sufficient magnitude, to apply. However, what can be generated easily are accurate angles by utilizing common stepper motors. These motors are capable of providing highly accurate angles when rotated, which can be used in the sequel as reference to the gyroscope. By rewriting equation (5) in terms of the integral, over the current measurement set, as:

$$
\begin{aligned}
\int \boldsymbol{\omega}_{m} d t & =\int \boldsymbol{T}_{\omega} \boldsymbol{\omega}_{\text {true }}+\boldsymbol{T}_{s} \boldsymbol{a}_{s}+\boldsymbol{\omega}_{b} d t, \\
\boldsymbol{\theta}_{m} & =\boldsymbol{T}_{\omega} \boldsymbol{\theta}_{\text {true }}+\boldsymbol{T}_{s} \int \boldsymbol{a}_{s} d t+\boldsymbol{\theta}_{b},
\end{aligned}
$$

where the norm of $\boldsymbol{\theta}_{\text {true }}$ can be chosen by rotating the motor to any desired angle, the overall problem is simplified down to the same problem as the accelerometer calibration, when excluding the acceleration sensitivity $\boldsymbol{T}_{s}$. The addition of $\boldsymbol{T}_{s}$ can however be isolated, as it will be visible in the gyroscope's bias when the sensor is at rest at different orientations, which implies that $\boldsymbol{T}_{s}$ is observable during the collection of the accelerometer's dataset, while $\boldsymbol{T}_{\omega}$ can be estimated from the rotating dataset.

\section{B. Measurement selection and design}

In contrary to [9] that used random orientations, the orientations were chosen in such a way that each axis had maximum excitation, plus all positive and negative permutations of the combinations of the axises were included, which gives 26 different orientations spread uniformly over the sphere. This approach has been adapted in order to achieve a full excitation of the measurements and thus allowing each matrix to be estimated.

\section{Optimization problem}

For numerically solving the calibration problem, and as the norms for $\boldsymbol{a}_{s}$ and $\boldsymbol{\theta}_{\text {true }}$ are known, the problem can be rewritten in the inverse models as:

$$
\begin{aligned}
& \hat{\boldsymbol{a}}_{i, s}=\underbrace{\hat{\boldsymbol{T}}_{a}^{-1}}_{\hat{\boldsymbol{T}}_{A}} \boldsymbol{a}_{i, m} \underbrace{-\hat{\boldsymbol{T}}_{a}^{-1} \hat{\boldsymbol{a}_{b}}}_{\hat{\boldsymbol{a}}_{B}}=\hat{\boldsymbol{T}}_{A} \boldsymbol{a}_{i, m}+\hat{\boldsymbol{a}}_{B}
\end{aligned}
$$

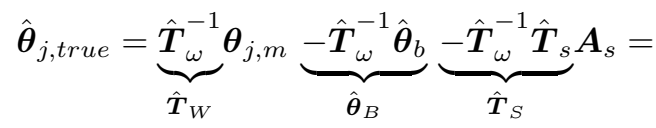

$$
\begin{aligned}
& =\hat{\boldsymbol{T}}_{W} \boldsymbol{\theta}_{j, m}+\hat{\boldsymbol{\theta}}_{B}+\hat{\boldsymbol{T}}_{S} \boldsymbol{A}_{j, s},
\end{aligned}
$$

where $\boldsymbol{A}_{j, s}=\int_{j} \hat{\boldsymbol{a}}_{s} d t$ is over the $j^{t h}$ measurement set, from which the following two nonlinear least-squares problems arise:

$$
\begin{array}{ll}
\underset{\hat{\boldsymbol{T}}_{A}, \hat{\boldsymbol{a}}_{B}}{\operatorname{minimize}} & \sum_{i}\left(\left\|\hat{\boldsymbol{a}}_{i, s}\right\|-\left\|\boldsymbol{a}_{s}\right\|\right)^{2} \\
\text { subject to } & s_{i, a}>0, \text { for } i \in[1,2,3]
\end{array}
$$

and

$$
\begin{aligned}
& \underset{\hat{\boldsymbol{T}}_{W}, \hat{\boldsymbol{\theta}}_{B}, \hat{\boldsymbol{T}}_{S}}{\operatorname{minimize}} \sum_{j}\left(\left\|\hat{\boldsymbol{\theta}}_{j, \text { true }}\right\|-\left\|\boldsymbol{\theta}_{\text {true }}\right\|\right)^{2}, \\
& \text { subject to } s_{i, \omega}>0 \text {, for } i \in[1,2,3]
\end{aligned}
$$

This problem will be solved in a 2-step approach, as equation (12) is dependent on the solution of equation (11). More specifically, to solve each of these nonlinear least-squares problems a Levenberg-Marquardt (LM) solver [10], [11] was leveraged.

\section{EXPERIMENTAL EVALUATION}

\section{A. Experimental setup}

The experimental setup presented in Figure 1 was designed and manufactured at Luleå University of Technology, where for the structural elements 3D printing was utilized. As presented if the aforementioned Figure, the calibration rig consists of two servo actuators, used to rotate the IMU board to the desired orientation, mounted on a stepper motor, which is used to rotate the board to an exact angle during the gyroscope calibration. The servos were chosen due to their simplicity and low weight for achieving a desired orientation, where the multitude of orientations, discussed in Section III$\mathrm{B}$, needs to be achieved approximately, as long as each axis receives ample excitation. 


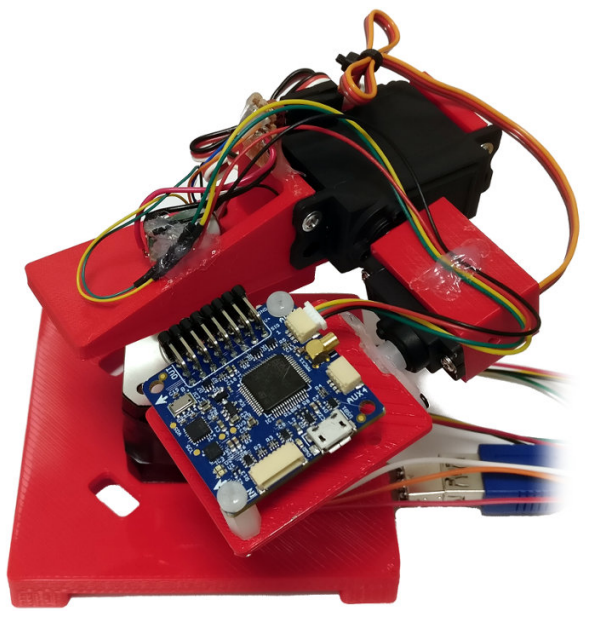

Fig. 1. An overview of the calibration rig with the IMU board mounted. All red parts are 3D printed and the two servo actuators used to rotate the IMU to desired positions are visible, while the stepper motor rotating the IMU is hidden under the IMU board.

To achieve a smooth and steady rotation from the stepper motor a Silent Step-Stick driver board, which has interpolated 1/256 micro stepping and voltage control over the motor coils, was utilized together with an Arduino Pro Micro ${ }^{1}$ which calculated acceleration and velocity curves together with control signals for the servo actuators, to minimize vibrations from the rig during the gyroscope calibration. The Arduino was controlled from a calibration node implemented in the Robotic Operating System (ROS) [12], where the maximum speed, acceleration and number of rotations of the stepper motor were runtime settable parameters.

The MEMS IMU utilized in the experiment was, the now classical, Invensense's MPU-6050 Accelerometer and Gyroscope System on a Chip (SOC) [13] for measuring acceleration and angular rate, with a full scale range of $\pm 16 \mathrm{~g}$ and \pm 2000 degrees/s, both with 16-bit accuracy. The sensor was configured to sample at $200 \mathrm{~Hz}$, where the data-ready signal was timestamped with 100 nanosecond accuracy, which was used as sampling clock, moreover the sensor is able to communicate with the Micro Controller Unit (MCU) via an $\mathrm{I}^{2} \mathrm{C}$ bus running at $400 \mathrm{kbit} / \mathrm{s}$. Finally, the whole experiment was conducted with a slight airflow over the IMU to keep it at a stable room temperature.

\section{B. Calibration results}

To apply the solver, a gravitational constant and a reference angle is needed. In the indicative experiments the choice was made to calibrate the accelerometer to 1 , which corresponds to $1 \mathrm{~g}$, while for the gyroscope the reference rotation was 4 revolutions, or $8 \pi$ radians.

The datasheet values [13] for the accelerometer indicates a sensitivity of $1 / 2048=0.00048828$ for $\hat{\boldsymbol{T}}_{A}$, while it predicts $\pi / 180 / 16.4=0.0010642$ for the sensitivity of $\hat{\boldsymbol{T}}_{W}-$ which are used as starting guesses for the optimization while $\hat{\boldsymbol{T}}_{S}$ was initialized to zero.

\footnotetext{
${ }^{1}$ https://www.sparkfun.com/products/12640
}
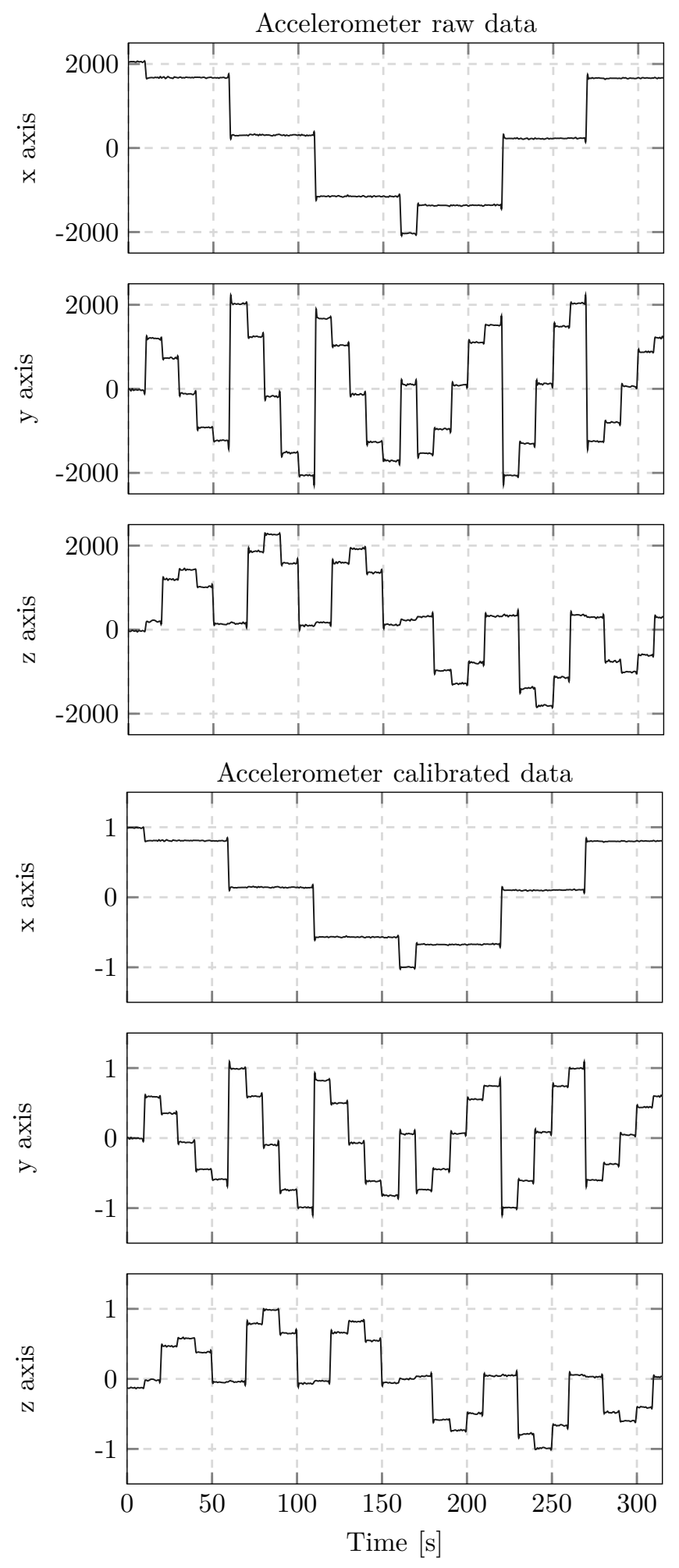

Fig. 2. The uncalibrated acceleration data from the experiment.

1) Full calibration: When applying the aforementioned calibration algorithm on the datasets in the first half of 

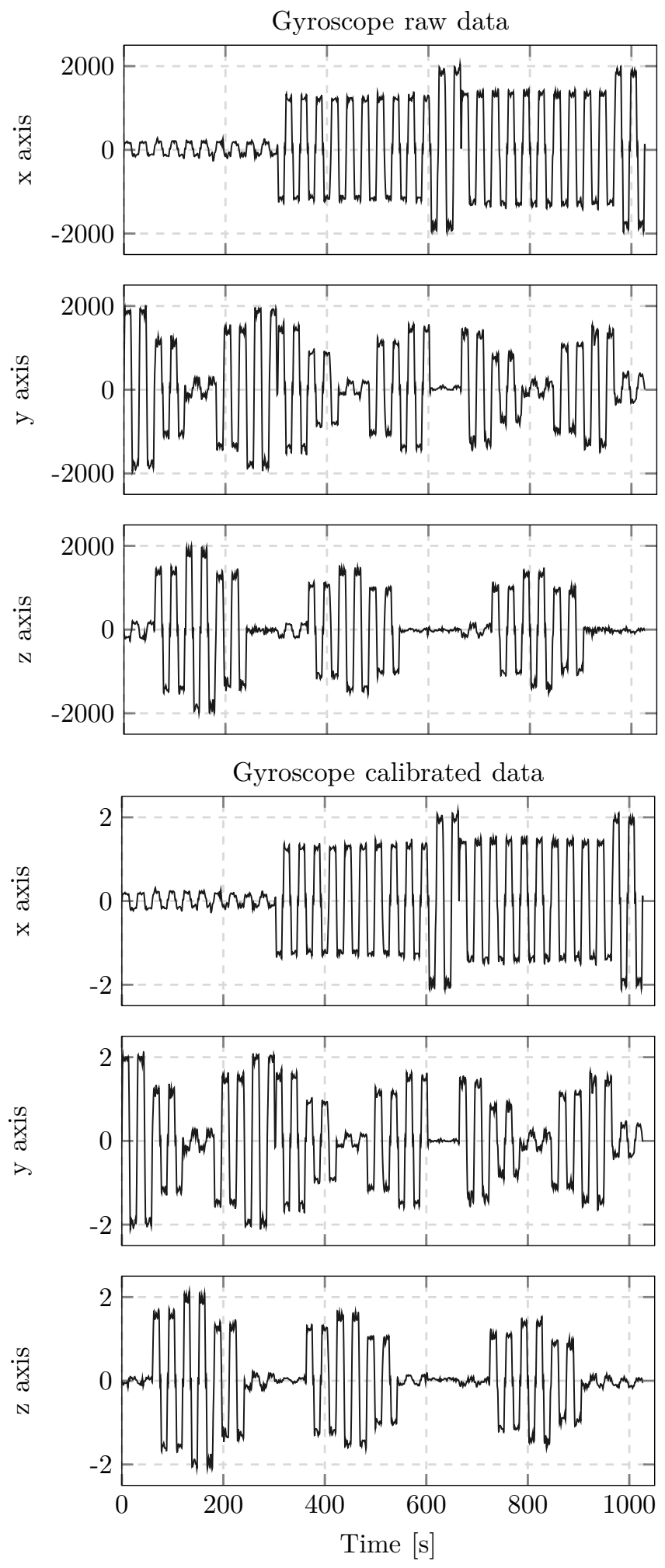

Fig. 3. The uncalibrated gyroscope data from the experiment.

Figure 2 and 3, the following resulting matrices are produced:

$$
\hat{\boldsymbol{T}}_{A}=\left[\begin{array}{ccc}
0.00048721 & 6.0916 \cdot 10^{-7} & 7.4076 \cdot 10^{-7} \\
-5.997 \cdot 10^{-7} & 0.00048529 & -8.8196 \cdot 10^{-6} \\
-2.0912 \cdot 10^{-7} & -4.2625 \cdot 10^{-8} & 0.00048447
\end{array}\right]
$$

$$
\begin{gathered}
\hat{\boldsymbol{T}}_{W}=\left[\begin{array}{ccc}
0.0010656 & 1.2485 \cdot 10^{-5} & 6.966 \cdot 10^{-5} \\
-1.34 \cdot 10^{-5} & 0.0010743 & 2.7523 \cdot 10^{-5} \\
-1.737 \cdot 10^{-5} & 6.9717 \cdot 10^{-5} & 0.0010716
\end{array}\right], \\
\hat{\boldsymbol{T}}_{S}=\left[\begin{array}{ccc}
0.00019684 & -0.0068028 & 0.030739 \\
0.0056904 & 1.6035 \cdot 10^{-5} & -0.003701 \\
-0.03198 & 0.0037953 & -0.00080715
\end{array}\right], \\
\hat{\boldsymbol{a}}_{B}=\left[\begin{array}{c}
-0.0086744 \\
0.010245 \\
-0.11256
\end{array}\right], \\
\hat{\boldsymbol{\theta}}_{B}=\left[\begin{array}{c}
-0.48227 \\
-0.57248 \\
0.26008
\end{array}\right],
\end{gathered}
$$

while when applying the calibration matrices, the second half of Figure 2 and 3 are produced together with the resulting norms in Figure 4 and 5. The gain and axis misalignment errors are presented in Table I. As it is being indicated there, the accelerometer and gyroscope shows gain errors of almost $1 \%$, while the gyroscope has a worst case axis misalignment error of $3.44^{\circ}$. The $\hat{\boldsymbol{T}}_{S}$ matrix clearly indicates the new trend in MEMS gyroscopes, with a maximum acceleration sensitivity of $31 \mathrm{mrad} / \mathrm{g}$, and as manufacturing processes and quality improve, these values are expected to approach 0 .

TABLE I

COLLECTION OF SENSOR ERRORS.

\begin{tabular}{|ccc} 
& Accelerometer & Gyroscope \\
\hline x sensitivity error & $0.22 \%$ & $0.13 \%$ \\
y sensitivity error & $0.60 \%$ & $0.95 \%$ \\
z sensitivity error & $0.78 \%$ & $0.92 \%$ \\
maximum axis & $1.08^{\circ}$ & $3.44^{\circ}$ \\
\hline misalignment & & \\
\hline
\end{tabular}

2) Gain only vs. Axis misalignment calibration: To depict the difference of performing full axis misalignment calibration, the off-diagonal elements in $\hat{\boldsymbol{T}}_{A}$ were set to 0 , and the result is presented in Figure 4. As it can be observed from this Figure, when considering the axis misalignment calibration the resulting norm does not exhibit the excessive peaks as with the gain-only calibration, clearly showing the impact of the full calibration.

3) Final remarks: From the calibration experiments it is evident that the angle based scheme is providing good results, while being simple to implements. The accuracy of the calibration, as seen in the norm Figures, is solid with minimal change from the nominal norms.

\section{CONCLUSions}

In this article a reduced complexity calibration method for MEMS IMUs have been presented, which does not need the rotating reference tables that are commonly used in gyroscope calibration. In this work fixed angle rotations have been utilized to observe the integral of the gyroscope data 

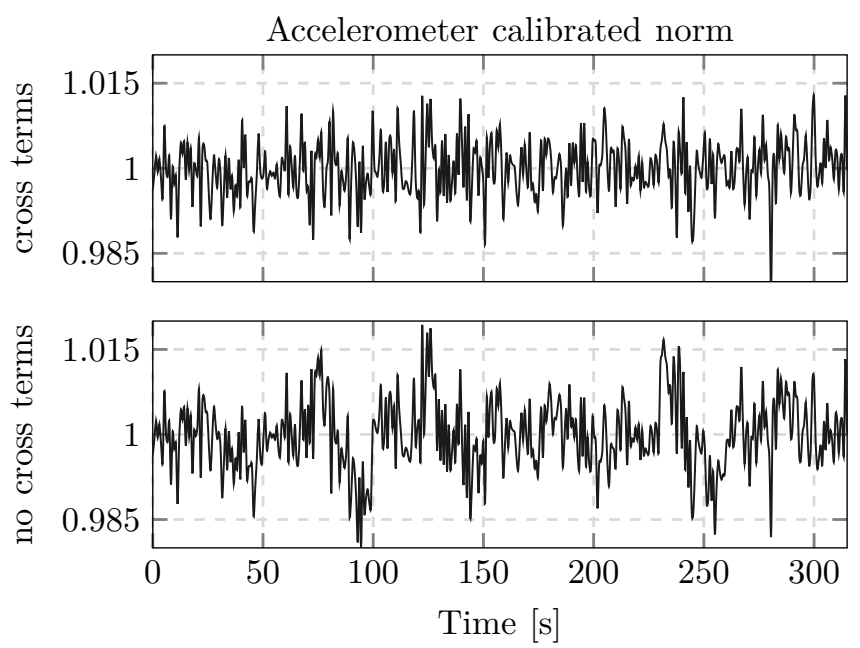

Fig. 4. The calibrated acceleration norm from the experiment in two cases, the top plot depicts the full calibration while the bottom plot depicts the gain-only calibration.

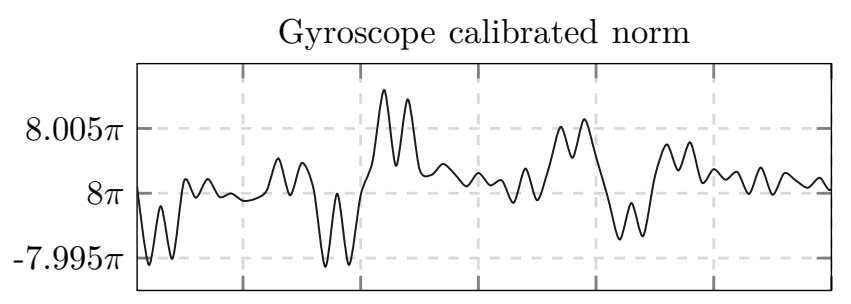

Fig. 5. The calibrated gyroscope norm from the experiment.

to find the corresponding calibration matrices, this has the significant merit of easiness of use, low cost and simplicity of construction, allowing anyone with basic electronics and embedded programming knowledge to calibrate an IMU.

\section{REFERENCES}

[1] J. Solà, "Quaternion kinematics for the error-state KF," Mar. 2015, working paper or preprint. [Online]. Available: https://hal.archivesouvertes.fr/hal-01122406

[2] S. Lynen, M. Achtelik, S. Weiss, M. Chli, and R. Siegwart, "A Robust and Modular Multi-Sensor Fusion Approach Applied to MAV Navigation," in Proc. of the IEEE/RSJ Conference on Intelligent Robots and Systems (IROS), 2013.

[3] N. Trawny and S. I. Roumeliotis, "Indirect Kalman Filter for 3D Attitude Estimation," Department of Computer Science \& Engineering, Tech. Rep., 2005.

[4] M. Bloesch, S. Omari, M. Hutter, and R. Siegwart, "Robust visual inertial odometry using a direct ekf-based approach," in Intelligent Robots and Systems (IROS), 2015 IEEE/RSJ International Conference on. IEEE, 2015, pp. 298-304.

[5] A. I. Mourikis and S. I. Roumeliotis, "A multi-state constraint Kalman filter for vision-aided inertial navigation," in Proceedings of the IEEE International Conference on Robotics and Automation (ICRA), Rome, Italy, April 10-14 2007, pp. 3565-3572.

[6] E. Fresk, G. Nikolakopoulos, and T. Gustafsson, "A Generalized Reduced-Complexity Inertial Navigation System for Unmanned Aerial Vehicles," IEEE Transactions on Control Systems Technology, vol. PP, no. 99 , pp. 1-16, 2016.

[7] D. Titterton and J. L. Weston, Strapdown inertial navigation technology. IET, 2004, vol. 17.

[8] M. Li, H. Yu, X. Zheng, and A. I. Mourikis, "High-fidelity sensor modeling and self-calibration in vision-aided inertial navigation," in 2014 IEEE International Conference on Robotics and Automation (ICRA). IEEE, 2014, pp. 409-416.
[9] I. Frosio, F. Pedersini, and N. A. Borghese, "Autocalibration of MEMS accelerometers," IEEE Transactions on Instrumentation and Measurement, vol. 58, no. 6, pp. 2034-2041, 2009.

[10] K. Levenberg, "A method for the solution of certain non-linear problems in least squares," Quarterly of applied mathematics, vol. 2, no. 2, pp. 164-168, 1944.

[11] D. W. Marquardt, "An algorithm for least-squares estimation of nonlinear parameters," Journal of the society for Industrial and Applied Mathematics, vol. 11, no. 2, pp. 431-441, 1963.

[12] M. Quigley, K. Conley, B. P. Gerkey, J. Faust, T. Foote, J. Leibs, R. Wheeler, and A. Y. Ng, "ROS: an open-source Robot Operating System," in ICRA Workshop on Open Source Software, 2009.

[13] Invensense MPU-6050 6-axis Motion Tracking device, https://www.invensense.com/products/motion-tracking/6-axis/mpu6050. 